\title{
ADAMTS13 is expressed in hepatic stellate cells
}

\author{
Wenhua Zhou ${ }^{1}$, Mari Inada ${ }^{2,3}$, Tai-Ping Lee ${ }^{4, *}$, Daniel Benten ${ }^{2}$, Sergey Lyubsky ${ }^{5}$, \\ Eric E Bouhassira ${ }^{1,6}$, Sanjeev Gupta ${ }^{2,7}$ and Han-Mou Tsai ${ }^{1}$ \\ ${ }^{1}$ Division of Hematology, Albert Einstein College of Medicine and Montefiore Medical Center, Bronx, NY, \\ USA; ${ }^{2}$ Marion Bessin Liver Research Center, Department of Medicine, Albert Einstein College of Medicine, \\ Bronx, NY, USA; ${ }^{3}$ Department of Medicine and Clinical Oncology, Graduate School of Medicine, Chiba \\ University, Japan; ${ }^{4}$ Department of Medicine, Veterans Affairs Medical Center, Northport, NY, USA; \\ ${ }^{5}$ Department of Pathology and Laboratory Medicine, Veterans Affairs Medical Center, Northport, NY, USA; \\ ${ }^{6}$ Department of Cell Biology, Albert Einstein College of Medicine, Bronx, NY, USA and ${ }^{7}$ Department of \\ Pathology, Albert Einstein College of Medicine, Bronx, NY, USA
}

\begin{abstract}
ADAMTS13 is a circulating zinc metalloprotease that cleaves the hemostatic glycoprotein von Willebrand factor (VWF) in a shear-dependent manner. Deficiency in ADAMTS13, owing to genetic mutations or autoimmune inhibitors, causes thrombotic thrombocytopenic purpura (TPP). Northern blot analysis has shown that ADAMTS13 is expressed primarily in the liver. By using real-time RT-PCR, we confirmed that in mice the liver had the highest level of the ADAMTS13 transcript. To identify the liver cell-type-specific origin of ADAMTS13, we used in situ hybridization techniques to investigate the pattern of ADAMTS13 expression in the liver; analyzed the ADAMTS13 proteolytic activity in the culture media of fractionated liver cells; and confirmed ADAMTS13 expression with RT-PCR analysis and cloning of the mouse ADAMTS13 gene. The results revealed that ADAMTS13 was expressed primarily in cell fractions enriched in hepatic stellate cells. The mouse ADAMTS13 cloned from primary hepatic stellate cells was similar to its human counterpart in digesting VWF and was susceptible to suppression by EDTA or the IgG inhibitors of patients with TTP. Since hepatic stellate cells are believed to play a major role in the development of hepatic fibrosis and cirrhosis, the identification of the liver cell-type expressing ADAMTS13 will have important implications for understanding pathophysiological mechanisms regulating ADAMTS13 expression.
\end{abstract}

Laboratory Investigation (2005) 85, 780-788, advance online publication, 4 April 2005; doi:10.1038/labinvest.3700275

Keywords: ADAMTS13; hepatic stellate cell; von Willebrand factor; metalloprotease

The von Willebrand factor (VWF) is a multimeric glycoprotein that mediates adhesion and aggregation of platelets at sites of vascular injury. A metalloprotease cleaves VWF at the Tyr1605Met1606 bond, generating homodimers of the 176 and $140 \mathrm{kDa}$ fragments. ${ }^{1,2}$ The VWF-cleaving protease is essential for preventing platelet aggregation in the circulation, and deficiency of the protease is associated with thrombotic thrombocytopenic purpura (TTP), a disease characterized by the development of VWF-platelet-rich thrombi in the arterioles

Correspondence: Dr H-M Tsai, MD, Division of Hematology, Montefiore Medical Center, 111 East 210th Street, Bronx, NY 10467, USA.

E-mail: htsai@montefiore.org

${ }^{*}$ Current address: Department of Medicine, North Shore University Hospital, Manhasset, NY, USA

Received 2 December 2004; revised and accepted 14 February 2005; published online 4 April 2005 and capillaries. ${ }^{3}$ Recent studies identified this protease as ADAMTS13, a member of the reprolysin-type zinc metalloprotease family. ${ }^{4-6}$

The human ADAMTS13 gene, spanning $37 \mathrm{~kb}$ on human chromosome 9q34, comprises 29 exons that encode a polypeptide of 1427-amino-acid residues and possibly several splicing isoforms. Although it shares with other members of the ADAMTS family a common domain architecture consisting of metalloprotease, disintegrin-like sequence, thrombospondin type 1 repeat, cysteine-rich and spacer regions, ADAMTS13 exhibits several distinct features, such as an RGDS sequence in the spacer domain and two copies of CUB domains at the carboxyl terminus. Substitution of the D residue in the RGDS sequence does not appear to diminish the proteolytic activity of ADAMTS13. ${ }^{7}$ Unlike other ADAMTS proteases, pro-ADAMTS13 is proteolytically active. ${ }^{8}$ These unique features of ADAMTS13 are consistent with 
the early phylogenetic divergence of the protease from other members of this recently recognized zinc metalloprotease family. ${ }^{9}$

In circulating blood, ADAMTS13 is enzymatically active. Similarly, transfection studies using cultured cells showed that ADAMTS13 was released in culture medium in an active form. Studies of patients with TTP reveal that plasma ADAMTS13 activity inversely correlates with the severity of TTP. ${ }^{10}$ Low ADAMTS13 activity levels have been described in patients with several conditions, including liver disease and sepsis. ${ }^{11-13}$ Nevertheless, the mechanisms for the decreased ADAMTS13 activity levels in these conditions are not clear and except for circulating inhibitors or mutations affecting the ADAMTS13 gene, the factors regulating plasma ADAMTS13 levels remain poorly understood. The lack of characterization of the cells that synthesize ADAMTS13 has limited the progress of investigation. Previous studies using Northern blot analysis demonstrated that full-length ADAMTS13 is expressed primarily in the liver. ${ }^{4-6}$ In this study, we determined the type of cells in the liver that express ADAMTS13.

\section{Materials and methods}

\section{Animals}

FVB/N mice were from Jackson Laboratories (Bar Harbor, ME, USA). The animals were maintained under controlled light and temperature conditions with free access to pelleted food and water. The Animal Care and Use Committees approved animal protocols in accordance with institutional and $\mathrm{NIH}$ guidelines.

\section{RNA In Situ Hybridization}

Human liver tissues, preserved in 4\% formaldehyde in phosphate-buffered saline solution (its usually $10 \%$ buffered formalin) after either autopsy or needle biopsy, were used for the study. The liver tissue blocks were sectioned at $4 \mu \mathrm{m}$ in thickness with a microtome and used in in situ hybridization using the procedures as previously described. ${ }^{14}$ To generate probes for hybridization, an ADAMTS13 cDNA from human fetal liver cDNA library spanning nucleotide 541-1680 and inserted in pBSIISK + , kindly provided by David Ginsburg (Howard Hughes Medical Institutes, Ann Arbor, MI, USA) was used as the template. The plasmid was used to prepare labeled antisense or sense RNA by in vitro transcription with bacteriophage T7 or T3 RNA polymerase in the presence of $12 \mu \mathrm{mol} / \mathrm{l}$ UTP and $4 \mu \mathrm{mol} / \mathrm{l}\left[{ }^{33} \mathrm{P}\right] \mathrm{UTP}$. The radiolabeled probe was used at $0.5-1.0 \times 10^{7}$ counts per minutes per slide. Slides were exposed for 2-3 weeks, counterstained with hematoxyline, and examined by both bright- and dark-field light microscopy.

\section{Cell Lines}

THLE-3 cell line (ATCC, cat. no. CRL-2706, Rockville, MD, USA), derived from primary normal human liver cells following introduction of the SV40 large $\mathrm{T}$ antigen, was propagated in culture dishes or flasks coated for $2 \mathrm{~h}$ at $37^{\circ} \mathrm{C}$ with $1 \% \mathrm{CELL}$ PRIME 100 (Cohesion, Palo Alto, CA, USA) using BEBM medium (Clonetics Corporation, Walkersville, MD, USA) and $10 \%$ fetal bovine serum (FBS). Human hepatocellular carcinoma-derived cell line, $\mathrm{HuH}-7$, and rat hepatic stellate cell lines CFSC-3H and -8B, which were originally created from Wistar male albino rats treated with $\mathrm{CCl}_{4}$ in the laboratory of Marcos Rojkind (Albert Einstein College of Medicine, Bronx, NY, USA; presently Walter Reed Army Medical Center, Washington, DC, USA), were maintained in Dulbecco's-modified Eagle's Medium (DMEM) (GIBCO/BRL, Gaithersburg, MD, USA) supplemented with $100 \mathrm{U} / \mathrm{ml}$ penicillin, $100 \mathrm{U} / \mathrm{ml}$ streptomycin and $10 \%$ heat inactivated FBS (GIBCO/BRL)). ${ }^{15,16} \mathrm{~A}$ hepatic stellate cell line, generated from primary normal human liver cells transfected with human telomerase reverse transcriptase (hTERT-HSC), was a generous gift of David Brenner (University of North Carolina, Chapel Hill, NC, USA; presently Columbia University, NY, USA). ${ }^{17}$ These cells were maintained in DMEM supplemented with 10\% FBS and $400 \mu \mathrm{g} / \mathrm{ml}$ geneticin (GIBCO/BRL) in $5 \% \mathrm{CO}_{2}$-humidified atmosphere at $37^{\circ} \mathrm{C}$.

For detecting ADAMTS13 release, $5 \mathrm{ml}$ serum-free culture media were conditioned with confluent cell monolayers in $25 \mathrm{~cm}^{2}$ flasks for $48 \mathrm{~h}$. After centrifugation to remove insoluble elements, conditioned culture media were concentrated 15 folds on Centricon concentrators (Millipore Corp, Bedford, MA, USA).

\section{Fractionation of Mouse Liver Cells}

Mouse liver was digested by collagenase perfusion as described previously. ${ }^{18}$ Briefly, the FVB/N mouse liver was perfused with Leffert's buffer followed by the same buffer containing $2 \mathrm{mmol} / \mathrm{l} \mathrm{CaCl}_{2}$ and $0.33 \mathrm{mg} / \mathrm{ml}$ collagenase. Then the liver was excised and filtered with an $80-\mu \mathrm{m}$ nylon gauze. Hepatocytes were pelleted by centrifugation under $50 \mathrm{~g}$ and plated in plastic culture dishes. After another round of centrifugation under $50 \mathrm{~g}$, the supernatant was pelleted under $350 \mathrm{~g}$ and applied to a two-layer discontinuous Percoll gradient. After centrifugation under $900 \mathrm{~g}$ for $20 \mathrm{~min}$, hepatic stellate cell and endothelial cell fractions were recovered from the upper and lower bands, respectively. The cell viability was tested by trypan blue dye exclusion and $1-2 \times 10^{6}$ cells were plated in 35-mm culture dishes. Hepatic stellate cells and hepatocytes were cultured in DMEM supplemented with 10\% FBS and antibiotics. The hepatocyte culture medium was 
supplemented with $5 \mu \mathrm{g} / \mathrm{ml}$ insulin and $5 \mu \mathrm{mol} / \mathrm{l}$ hydrocortisone (Sigma Chemical Co., St Louis, MO, USA). Endothelial cells were cultured in 199 medium supplemented with $20 \%$ FBS and antibiotics (GIBCO/BRL).

\section{Cytostainings for Characterization of Primary Mouse Cells}

Glycogen staining followed a previously described protocol. ${ }^{19}$ For CD31 immunostaining, cell smears were air-dried and fixed in cold acetone for $10 \mathrm{~min}$ followed by incubation with rat anti-mouse CD31 (BD Biosciences Pharmingen, San Diego, CA, USA) diluted $1: 100$ in $3 \%$ goat serum for $1 \mathrm{~h}$ at room temperature. For macrophage/Küpffer cell marker F4/80, cells were fixed with $4 \%$ paraformaldehyde in PBS for $10 \mathrm{~min}$ and incubated with rat anti-mouse F4/80 (1:500, Serotec, Raleigh, NC, USA). Antibody binding was detected by incubation with anti-rat IgG labeled with Alexa Fluor 546 (1:500, Molecular Probes) for $1 \mathrm{~h}$ at RT. After washing with PBS, cells were counterstained with DAPI-Antifade (Molecular Probes, Eugene, OR, USA).

\section{Reverse Transcription and PCR}

Total RNA was isolated from tissues or cells by using the Total RNA Purification System (Invitrogen) and subjected to reverse transcription by using ThermoScript RT-PCR System for First-Strand cDNA Synthesis kit (Invitrogen). The ADAMTS13 sequence was amplified with PCR by using the nucleotide sequences $5^{\prime}$-atgagccagctttgcctgtg- $3^{\prime}$ and $5^{\prime}$-ctaggacagagccaggctgt- $3^{\prime}$ as the primers. The PCR products were further amplified by using the nested sequences $5^{\prime}$-tcatccttgatgagcgtctg- $3^{\prime}$ and $5^{\prime}$-aggaagccagggctaaactc- $3^{\prime}$ as the primers. The DNA bands were separated by agarose gel electrophoresis and visualized by staining with ethidium bromide. Their nucleotide sequences were determined at the DNA Sequencing Facility of the Albert Einstein College of Medicine using the Applied Biosystems model 3700 DNA Sequencer. For housekeeping controls, the sequences $5^{\prime}$-acccacactgtgcccatctac and $5^{\prime}$ gccatctcctgctcgaagtc were used as the primers to amplify $\beta$-actin.

\section{Real-Time RT PCR}

cDNA was prepared as in RT-PCR experiments. Realtime RT-PCR of ADAMTS13 was performed on a LightCycler (Roche Applied Science, Indianapolis, IN, USA), using the sequences $5^{\prime}$-tccttctaccactgggatgc- $3^{\prime}$ and $5^{\prime}$-ccatcatcctgaggaccact- $3^{\prime}$ as primers. The same sequences used in RT-PCR were used as the primers to amplify $\beta$-actin. The cycling condition was $50^{\circ} \mathrm{C}, 2 \mathrm{~min} ; 95^{\circ} \mathrm{C}, 3 \mathrm{~min}$; 50 cycles of $95^{\circ} \mathrm{C}$, $5 \mathrm{~s}, 58^{\circ} \mathrm{C}, 10 \mathrm{~s}$, and $72^{\circ} \mathrm{C}, 10 \mathrm{~s} ; 95^{\circ} \mathrm{C}, 30 \mathrm{~s} ; 55^{\circ} \mathrm{C}, 30 \mathrm{~s}$; $70^{\circ} \mathrm{C}, 0 \mathrm{~s} ; 95^{\circ} \mathrm{C}, 0 \mathrm{~s}$; and $40^{\circ} \mathrm{C} 1 \mathrm{~min}$. SYBR Green I was added to the reaction mixtures for tracking the generation of dsDNA. The intensity of fluorescence was measured at the end of each elongation step of the PCR reaction. The specificity of the PCR reactions was confirmed by analysis of the melting curves and in some instances also by agarose gel electrophoresis of the reaction products. We used serially diluted RNA samples from one randomly selected FVB/N mouse liver to construct the reference curves for ADAMTS13 and $\beta$-actin. The ADAMTS13 transcript level of each sample, as determined by the second derivative maximum method, was adjusted for the level of $\beta$-actin transcript and expressed as a multiple or fraction of the ADAMTS13 transcript level in the liver.

\section{Transient Expression of Mouse ADAMTS13 (mADAMTS13)}

The complete coding sequence of mADAMTS13 was amplified with the PfuUltra DNA polymerase (Stratagene, La Jolla, CA, USA), using the sequences $5^{\prime}$ aaagatgagccagctttgcC- $3^{\prime}$ and $5^{\prime}$-ctaggacagagccaggctgt$3^{\prime}$ as the primers. The PCR product was ligated into the mammalian expression vector pcDNA3.1/V5-His (Invitrogen). We transfected this plasmid into actively growing COS-7 cells with Lipofectamine 2000 (Invitrogen). At $48 \mathrm{~h}$, the conditioned serumfree medium was collected and concentrated 15-fold on Centricon YM-30 concentrators (Millipore, Billerica, MA, USA). The cells were lysed in an equal volume of SDS-PAGE sample buffer. Recombinant proteins were separated by $7.5 \%$ SDS-PAGE and visualized by immunoblotting with monoclonal anti-V5 antibody (Invitrogen), horseradish peroxidase-conjugated anti-mouse IgG, and SuperSignal chemiluminescent substrate (Pierce, Rockford, IL, USA).

\section{Determination of ADAMTS13 Activity Levels and Protein Concentrations}

The ADAMTS13 activity levels in either cell culture medium or plasma samples were determined by measuring the proteolytic fragments produced from the human VWF substrate, using previously described procedures. ${ }^{20}$ Plasma samples from patients with TTP were obtained as in previous studies. To determine the concentrations of recombinant ADAMTS13 proteins, we immunoblotted the protein with monoclonal anti-6His (Invitrogen), and obtained the protein concentration by reading the optic density against a concentration-optic density curve constructed from the corresponding values of a purified recombinant protein GST-1596VWF16686 His with a known protein concentration. ${ }^{21}$ All ADAMTS13 samples were stored at $-70^{\circ} \mathrm{C}$. The Institutional Review Boards approved the use of human liver and plasma samples. 


\section{Results}

Distribution of ADAMTS13 Transcripts in the Human Livers

We investigated the pattern of ADAMTS13 transcript expression by in situ hybridization in seven liver tissues: one without evidence of liver disease, two with chronic hepatitis $\mathrm{C}$, two with cirrhosis, and one each with metastatic colonic or bronchogenic adenocarcinoma. Examination of the liver sections hybridized with ${ }^{33}$ P-labeled ADAMTS13 RNA antisense probe under either bright- or darkfield light microscopy revealed that ADAMTS13 transcripts existed in aggregates perinuclearly primarily in a subset of liver cells (Figure 1). In the normal human liver, the ADAMTS13-positive cells appeared to be in perisinusoidal locations and displayed smaller, slightly elongated nuclei, with denser chromatin compared to hepatocytes (panels a-d). Such aggregates of deposits were not found in the control sections hybridized with a sense probe (not shown). Individual radioactivity granules were seen in sections hybridized with either antisense or sense probe, indicating that at least some of these granules represent nonspecific deposits. The overall pattern of ADAMTS13 expression was not different in liver sections with type $C$ viral hepatitis (panels e and $\mathrm{f}$ ), cirrhosis (panels g and h), or metastasis (not shown), except that ADAMTS13 cells were not present in the area of inflammatory infiltrates of hepatitis (panels e and f) but clustered adjacent to fibrous septae in the cirrhotic liver (panels $g$ and $h$ ). In all liver sections examined, no expression of ADAMTS13 was observed in cells situated within the portal tracts, the liver sinusoidal endothelial cells (LSEC), or within the areas occupied by metastatic cancer cells (not shown).

\section{ADAMTS13 Activity in the Culture Media of Cell Lines and Primary Liver Cells}

To determine whether hepatocytes or hepatic stellate cells (HSC) synthesized ADAMTS13, we analyzed conditioned cell culture medium from human hepatoma cell line HUH-7, human hepatocyte cell line THLE-3, human HSC line hTERT-HSC, and rat HSC CFSC-3H or $-8 \mathrm{~B}$ cells.

We also analyzed primary mouse liver cells fractionated into hepatocytes, stellate cells and endothelial cells fractions by differential centrifugation (see Methods). The hepatocyte fraction was highly pure and was not contaminated with CD31positive LSEC, F4/80-positive Küpffer cells, or vitamin A containing HSC (Figure 2, panels a-c). The LSEC fraction demonstrated over $70 \%$ LSEC with CD31-staining and only extremely rare hepatocytes or HSC $(<1 \%)$, although $5-15 \%$ cells showed F4/80-staining, indicating the presence of Küpffer cells (Figure 2, panels d-g). The HSC fraction contained cells with vitamin A autofluor- escence in over $50 \%$; however, this cell fraction contained up to $40 \%$ LSEC with CD31-staining and up to $15 \%$ Küpffer cells with F4/80-staining (Figure 2 , panels $\mathrm{h}-\mathrm{k}$ ), indicating a relatively heterogeneous cell population. All cell fractions, that is, hepatocyte, LSEC and HSC fractions were highly viable and monolayer cell cultures were successfully established (Figure 2, panels a, d and h).

Analysis of the culture media conditioned with human hepatocyte cell line THLE-3 or HUH-7, rat HSC line CFSC-3H or $-8 \mathrm{H}$, or human HSC line (hTERT-HSC) revealed that cells derived from HSC, but not cells derived from hepatocytes, released ADAMTS13. The results with CFSC-3H and THLE-3 are illustrated (Figure 3, panel a). These results were confirmed by analysis of the culture media conditioned with primary mouse liver cells: ADAMTS13 protease activity was detected in culture media conditioned with primary mouse HSC, but not in conditioned medium from either primary hepatocytes (Hep) or LSEC (Figure 3, panel b).

\section{Analysis of the ADAMTS13 Transcript by Real-Time RT-PCR}

To confirm that the liver is the major organ expressing ADAMTS13, we analyzed the levels of ADAMTS13 transcripts by real-time RT-PCR in various tissues of two $\mathrm{FVB} / \mathrm{N}$ mice and found that in both mice ADAMTS13 transcript was readily detected in the liver, but was either undetectable or detected at much lower levels in the spleen, lung, brain, peripheral blood cells, heart, or skeletal muscle (Figure 4).

\section{Analysis of ADAMTS13 Expression by RT-PCR}

To determine that mouse HSC expressed the fulllength mRNA of ADAMTS13, we amplified the cDNA generated from cell fractions containing mouse HSC, hepatocytes, or LSEC by using the primer sequences that encompassed the published complete coding sequence of mouse ADAMTS13 (NM_001001322), followed by another round of PCR with nested primers that amplified the sequence between nucleotides 451 and 4196. As demonstrated in Figure 5, panel a, the PCR experiments generated a 3.7-kb product from the whole mouse liver and the HSC fraction but not from the hepatocyte or LSEC fraction. Sequence analysis confirmed that the PCR product consisted of the expected mADAMTS13 (not shown).

\section{Cloning of Mouse Full-Length ADAMTS13}

The mouse ADAMTS13 produced by PCR was cloned in pcDNA3.1/V5-His. Sequence analysis confirmed the construct contained the complete coding sequence of $m A D A M T S 13$ gene, encoding a 

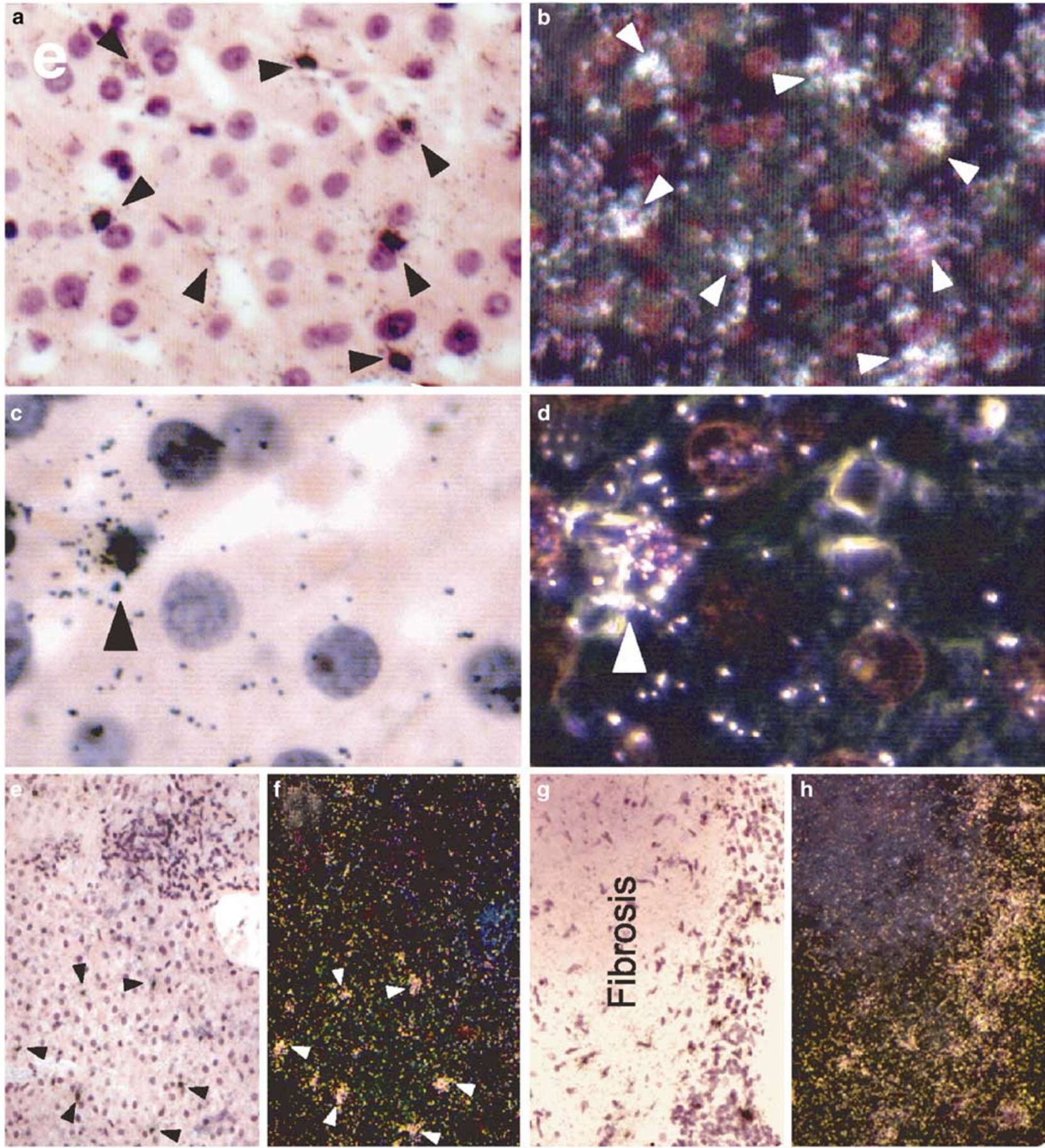

Figure 1 Digital light micrographs of adult human liver tissue sections hybridized with ADAMTS13 antisense RNA probe or sense RNA probe (not shown). Panels (a, c, e and $\mathbf{g}$ ) bright-field photos; panels (b, d, f, and $\mathbf{h}$ ) dark-field photos of the corresponding fields. (a and $\mathbf{c}$ ) ADAMTS13-positive cells (arrows) in a normal human liver, which are better visualized in the dark-field photos of the same fields (panels b and $\mathbf{d}$ ). (e and f) A section of liver with viral hepatitis C, showing ADAMTS13-positive cells (arrows) in the liver parenchyma, a pattern similar from that observed in the normal liver. ADAMTS13 cells are not present in the inflammatory infiltrate adjacent to a central vein. ( $(\mathbf{g}$ and $\mathbf{h}$ ) A section of a cirrhotic liver depicting the transition between a fibrous septum and the reactive cells, many of them expressing ADAMTS13. Original magnifications: panels (a and b) $\times 200$; panels $(\mathbf{c}$ and $\mathbf{d}) \times 400$; panels $(\mathbf{e}-\mathbf{h}) \times 100$.

protein of 1426 amino acid residues, coinciding with the published sequence data (NM_001001322). Expression of this construct in COS-7 cells resulted in the synthesis of a $190-\mathrm{kDa}$ protein, $12 \%$ of which was released in the culture medium (Figure 5, panel b). By comparing with a His-tagged reference protein, we determined that the concentration of the secreted protein was $8 \mathrm{nmol} / \mathrm{l}$. The protein 

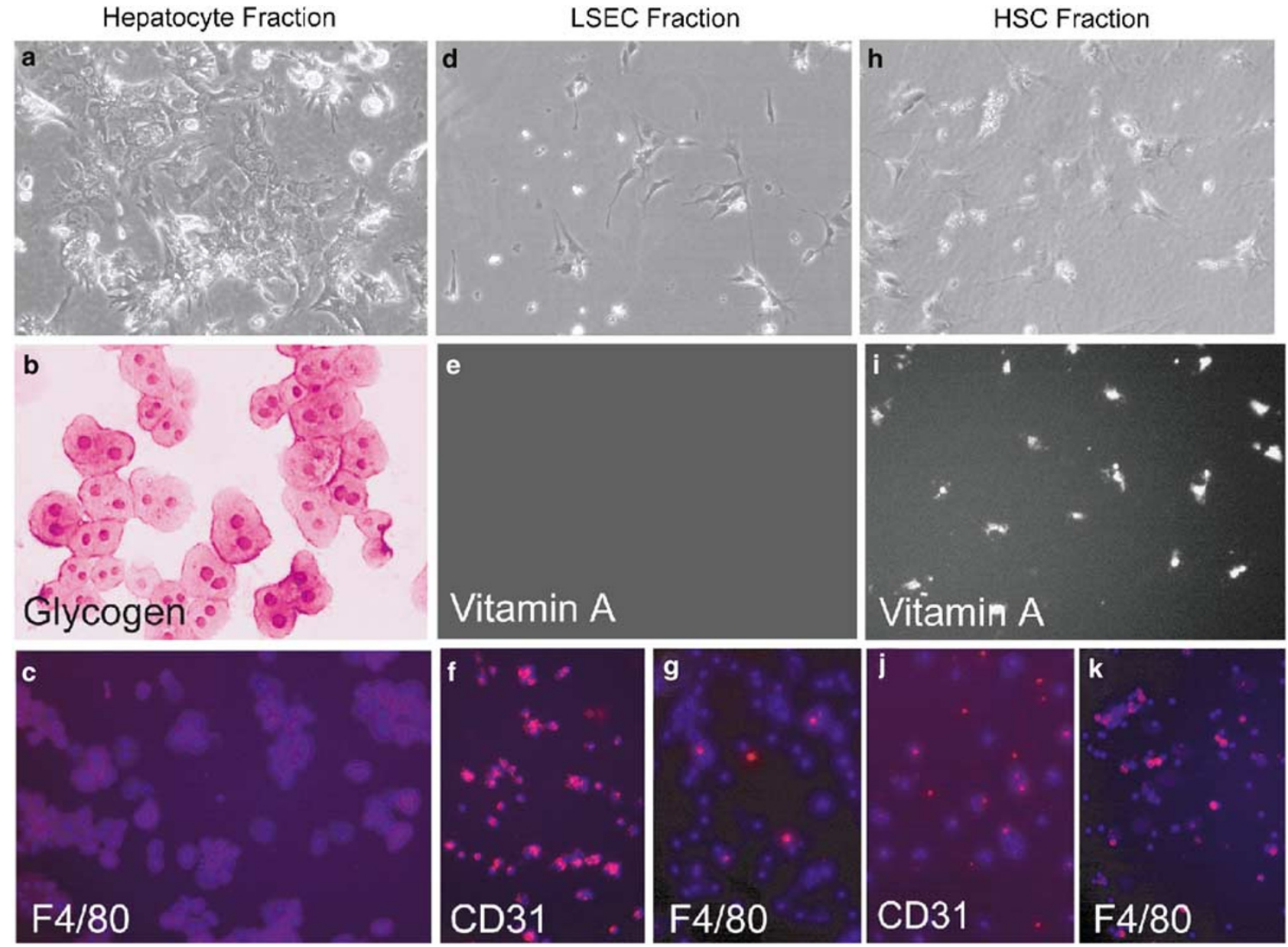

Figure 2 Selected properties of primary mouse liver cells, which were separated into fractions enriched in hepatocytes (a-c), LSEC (d-g) and HSC (h-k). The cell fractions were examined for hepatocyte marker (glycogen), endothelial marker (CD31), HSC marker (vitamin A autofluorescence), and Küpffer cell marker (F4/80), as indicated. Panels (c, f, g, j and k) were counterstained with DAPI (blue color). CD31 and F4/80 staining is seen in red color. Panels a, $\mathbf{d}$, and $\mathbf{h}$ show cells cultured for 4 days to indicate that isolated cells were healthy. Original magnification: $\times 400$.

cleaved hVWF substrate with $3 \mathrm{U} / \mathrm{ml}$ of proteolytic activity ( $1 \mathrm{U}$ equals the protease activity in $1 \mathrm{ml}$ of normal human plasma), generating from the hVWF substrate the same fragments expected of hADAMTS13 (Figure 5, panel c). The protease activity was suppressed by EDTA as well as by each of eight randomly selected TTP plasma samples, one of which is illustrated (Figure 5, panel d). The specific protease activity of recombinant mADAMTS13 ( $3 \mathrm{U} / \mathrm{ml} \div 8 \mathrm{nmol} / \mathrm{l}=375 \mathrm{U} / \mathrm{nmol}$ ) was similar to the presumed specific activity of human plasma ADAMTS13 $(1 \mathrm{U} / \mathrm{ml} \div 5 \mathrm{nmol} / \mathrm{l}=200 \mathrm{U} / \mathrm{nmol}$, assuming plasma ADAMTS13 level $=1 \mu \mathrm{g} / \mathrm{ml}$, or $5 \mathrm{nmol} /$ 1). ${ }^{22}$ These estimates need to be confirmed in the future by direct analysis of purified proteins. We also determined the ADAMTS13 activity level in the heparin-anticoagulated plasma of $\mathrm{FVB} / \mathrm{N}$ mice by using the same hVWF substrate and found that the mean ( \pm s.d.) plasma ADAMTS13 activity level was $3.09 \pm 0.45 \mathrm{U} / \mathrm{ml}$ in $14 \mathrm{FVB} / \mathrm{N}$ mice (age 12-16 weeks). However, since the ADAMTS13 protein concentration in mouse plasma has not been determined, the specific activity level of mouse plasma ADAMTS13 is unknown.

\section{Discussion}

To understand the factors affecting the level of ADAMTS13 in plasma, we have characterized in this study the cellular source of ADAMTS13, a member of the recently recognized ADAMTS superfamily. ${ }^{9}$ Our findings here indicate that HSC are the most active hepatic source of ADAMTS13 production. This result may provide the basis for future investigations to elucidate the regulation of ADAMTS13 in thrombotic thrombocytopenic purpura and various pathophysiological conditions.

Previous studies using Northern blot analysis demonstrated that ADAMTS13 is expressed primarily in the human liver. Nevertheless, experiments using RT-PCR detected the presence of ADAMTS13 
Cell Lines


b

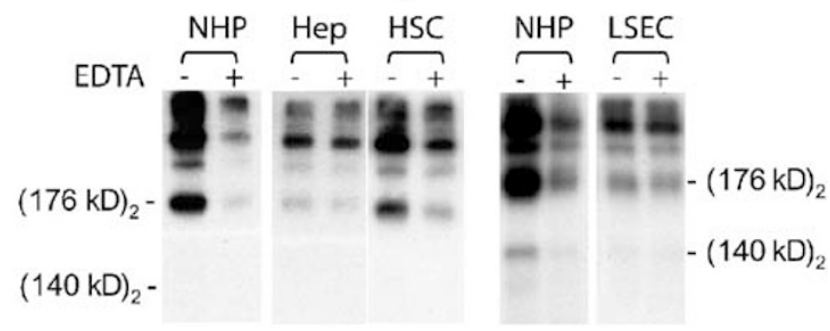

Figure 3 Detection of ADAMTS13 activity in the culture media of liver cells by SDS PAGE and immunoblotting. (a) ADAMTS13 activity was detected in the culture medium conditioned with a rat line CFSC-3H (HSC), but not with a hepatocyte cell line THLE3 (Hep). NHP: normal human plasma. (b) ADAMTS13 activity was detected in the culture medium of primary stellate cell fraction (HSC) but not in the primary hepatocytes (Hep) or LSEC of the mouse liver. To assay ADAMTS13 activity, a human VWF substrate was added to the test sample in the absence $(-)$ or presence $(+)$ of EDTA. In the absence of EDTA, the ADAMTS13 activity in normal human plasma (NHP) or HSC caused an increase in the homodimers of the $176-\mathrm{kDa}$ and the $140-\mathrm{kDa}$ fragments, labeled as $(176 \mathrm{kDa})_{2}$ and $(140 \mathrm{kDa})_{2}$ respectively. No such increase was detected in the culture media of hepatocytes or endothelial cell. The assay had a sensitivity of detecting a protease activity level at $10 \%$ of that in the normal control plasma.

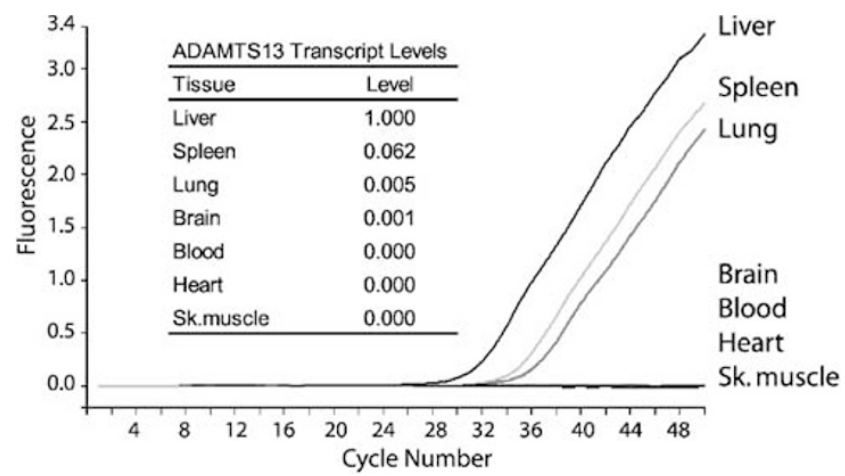

Figure 4 Analysis of mADAMTS13 transcript levels by RT-PCR. RNA molecules extracted from various tissues of FVB/N mice were reverse transcribed to cDNA and amplified by PCR. The levels of PCR products were traced by measuring the fluorescence intensity of CYBR Green I in a LightCycler. Representative tracings of one experiment are shown. For comparison among the tissues, the ADAMTS13 transcript level of each tissue was normalized for the $\beta$-actin level and expressed as a fraction of the corresponding ADAMTS13 level in the liver. The table shows the means of the values obtained from two mice. a

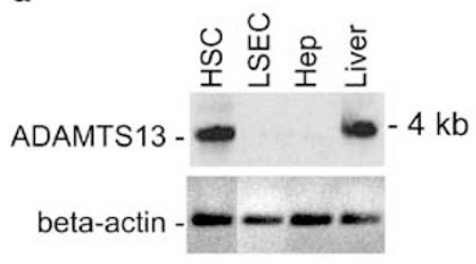

C

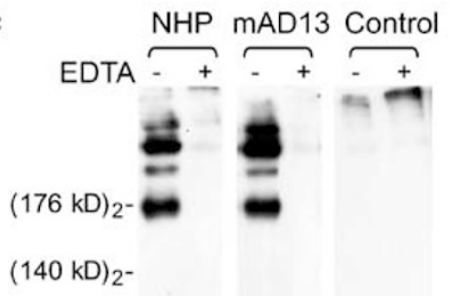

b
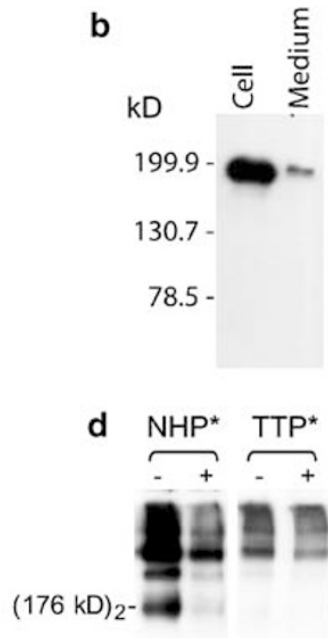

$(140 \mathrm{kD})_{2^{-}}$
Figure 5 RT-PCR and cloning of mouse ADAMTS13. (a) RT-PCR generated a 3.7-kb ADAMTS13 band from the RNA samples extracted from mouse liver or primary HSC. No PCR products were generated from primary hepatic endothelial cells (LSEC) or hepatocytes (Hep). As a result of the nesting design, the PCR product was smaller than the expected size of $4.7 \mathrm{~kb}$. (b) Transfection of COS-7 cells with a construct of full-length murine ADAMTS13 resulted in the synthesis of a 190-kDa protein, visualized on the immunoblot with anti-V5. Approximately $12 \%$ of the protein was released in the culture medium. (c) VWFcleaving activity of mouse ADAMTS13. Each sample was tested in the absence or presence of EDTA. An increase of the VWF fragments in the absence of EDTA resulted from digestion of the VWF substrate by ADAMTS13 in either normal human plasma (NHP) or mADAMTS13 transfection medium (mAD13). No protease activity was detected in the control medium conditioned with cells transfected with vector alone. Owing to its high proteolytic level, the recombinant mouse ADAMTS13 was diluted three folds before the analysis. (d) Inhibition of mADAMTS13 activity by TTP plasma. In the mixing study, an equal volume of a TTP patient plasma (TTP*) or normal plasma (NHP*) was added to normal control plasma before the ADAMTS13 activity levels in the mixtures were analyzed. The TTP plasma, but not the normal plasma, suppressed the mouse ADAMTS13 activity. Prior to the mixing study, both plasma samples had been heated at $56^{\circ} \mathrm{C}$ to inactivate endogenous ADAMTS13 activity $\left({ }^{*}\right)$. The result with one TTP plasma sample is shown. Similar suppression was observed with each of seven other TTP plasma samples tested.

transcript in a varieties of tissues, ${ }^{4-6,23}$ and one study describes the expression of ADAMTS13 in human platelets. ${ }^{24}$ However, the RT-PCR studies did not directly compare the levels of expression among the different tissues. In our real-time RT-PCR experiments, we designed the primers to amplify the portion of ADAMTS13 that is present in all the known isoforms, and the results confirm quantitatively that mADAMTS13 is expressed most actively in the liver. In the other tissues, the ADAMTS13 transcript is present at much lower levels or undetectable.

The in situ hybridization results revealed that hADAMTS13 transcript was not present throughout all liver cells. Instead, it was found only in a subset 
of liver parenchymal cells. The morphological features and anatomic location of cells expressing ADAMTS13 transcripts suggested that these were HSC. The cellular location of the diffuse radioactivity granules could not be ascertained morphologically. By comparing with the control sections of sense probes, it was apparent that some of the granules represented nonspecific deposits. On the other hand, since hepatic stellate cells have 3dimensional structures with elongated projections wrapping around the basal surface of endothelial cells, sections of these cytoplasmic projections are present throughout the liver that may appear unconnected to the cells. Therefore, we cannot exclude the possibility that this structural characteristic of HSC may account for some of the individual granules that do not appear to cluster around particular cells.

The morphological features suggest but do not prove that HSC express ADAMTS13. We performed further studies using cell lines and fractionated primary liver cells to confirm that HSC express ADAMTS13. Studies of cell lines revealed that ADAMTS13 protease activity was detected in the culture media of three HSC lines investigated, including one from the human liver and two from the rat liver, but was not detected in the culture media of hepatoma-derived HUH-7 or human hepatocyte-derived THLE-3 cell line.

Since immortalized cell lines may not fully represent the properties of primary cells, we additionally utilized primary mouse liver cells to analyze ADAMTS13 production. A recent study reported that certain mouse strains harbor an intracisternal-associated particle (IAP)-type retrotransposon in the ADAMTS13 gene, which leads to the expression of a truncated form of ADAMTS13. ${ }^{23}$ Therefore, in this study, we used the FVB/N mouse strain, which, based on our PCR analysis of genomic DNA, does not contain the IAP-type retrotransposon (data not shown).

Indeed, protease analysis detected VWF cleaving activity in culture media of the primary HSC fraction, but not of the hepatocyte or LSEC fractions. Furthermore, the RT-PCR experiments generated a full-length ADAMTS band from the FVB/N mouse liver and primary mouse HSC, but not from either primary mouse hepatocytes or LSEC. Also, we found that cloned constructs of the full-length ADAMTS13 cDNA expressed the ADAMTS13 protease that was capable of cleaving the hVWF substrate. Like its human counterpart, the recombinant mADAMTS13 was sensitive to suppression by cation chelator EDTA or IgG inhibitors of TTP patients and its specific activity level is very similar to or higher than that of the human plasma ADAMTS13. These similarities are not surprising since the $\mathrm{mA}$ DAMTS13 and its substrate VWF are both $70-80 \%$ identical to their human homologues. Banno et $a l^{23}$ cloned the ADAMTS13 from mouse livers and showed that the protease cleaved a VWF fragment of 73 amino-acid residues that spans the presumed Tyr1605-Met1606 cleavage site of the VWF protein.

Our cell isolation procedures produced enriched, albeit nonhomogeneous fractions of specific liver cell types. The hepatocyte fraction was most enriched and showed virtually no contamination with other cell types. On the other hand, the LSEC fraction contained significant numbers of Küpffer cells, and the HSC fraction contained significant numbers of LSEC, as well as Küpffer cells. Most procedures to separate liver cells take advantage of differences in cell density. We found that our cell separation procedures were convenient for the initial screen and produced reliable results of ADAMTS13 expression for the following reasons. First, the hepatocyte fraction and LSEC fraction were essentially negative for ADAMTS13 expression, leading to confidence that contamination of the HSC fraction with these cells would not have confounded our results. Second, we did not find ADAMTS13 expression in peripheral blood leukocytes, which contain circulating mononuclear cells that are the counterparts of Küpffer cells. Similarly, absence of ADAMTS13 expression in the LSEC fraction, despite up to $10 \%$ Küpffer cells in this fraction was against ADAMTS13 expression in Küpffer cells. Thirdly, ADAMTS13 was expressed in HSC-derived cell lines and not hepatocytederived cell lines. Therefore, we interpret our results to indicate that ADAMTS13 expression was a function of HSC, irrespective of the presence of additional cell types in this fraction.

HSC, which account for approximately $15 \%$ of all liver cells, are phenotypically heterogeneous, as indicated by variable intensity of vitamin A autofluorescence and expression of desmin, glial fibrillary acidic protein, or alpha smooth muscle actin. Further complicating laboratory studies, HSC vary in their biological functions, ranging from lipid and vitamin A storage, contractile activity capable of modulating hepatic vascular responses, cytokine release, and myofibroblast transformation during fibrosis. ${ }^{25}$ It is presently unknown how these phenotypic variations in HSC might affect the expression of ADAMTS13. Since our studies detect the presence of many ADAMTS13-positive cells surrounding the fibrous septae of cirrhotic liver, it is possible that ADAMTS13 is synthesized in activated HSC. In fact, both human and rat HSC lines used in this study exhibit features of activation. Further studies are needed to delineate how the activation state of HSC may affect the expression and synthesis of ADAMTS13.

In summary, the study suggests that the liver cells expressing ADAMTS13 are morphologically similar to HSC. This interpretation is further corroborated by the results from cell lines and primary liver cell fractions, demonstrating that HSC expresses the ADAMTS13 transcript and secretes active ADAMTS13. Additionally, ADAMTS13 cloned from mouse HSC is proteolytically active and exhibit 
features similar to human ADAMTS13. As a result of their contribution to fibrogenesis, the stellate cell is considered a main culprit of liver cirrhosis. ${ }^{26}$ The successful localization of the cell-type synthesizing ADAMTS13 will facilitate further studies to define the regulation of ADAMTS13 transcription, translation, and secretion under physiological and pathological conditions. These studies should offer novel insights into the pathogenesis and therapies of TTP.

\section{Acknowledgements}

This study was supported in part by Grants R01HL62136, R01HL72876 (HMT), R01DK46952 and P30-DK-41296 (SG) from the National Institutes of Health, and a postgraduate scholarship Grant BE2559/1-1(DB) from Deutsche Forschungsgemeinschaft. The authors thank Dr Katia Manova for assistance in performing in situ hybridization experiments and Dr Marcos Rojkind and David Brenner for providing the cell lines used in this study.

\section{References}

1 Dent JA, Berkowitz SD, Ware J, et al. Identification of a cleavage site directing the immunochemical detection of molecular abnormalities in type IIA von Willebrand factor. Proc Natl Acad Sci USA 1990;87:6306-6310.

2 Tsai HM, Sussman II, Nagel RL. Shear stress enhances the proteolysis of von Willebrand factor in normal plasma. Blood 1994;83:2171-2179.

3 Tsai HM. Advances in the pathogenesis, diagnosis, and treatment of thrombotic thrombocytopenic purpura. J Am Soc Nephrol 2003;14:1072-1081.

4 Levy GG, Nichols WC, Lian EC, et al. Mutations in a member of the ADAMTS gene family cause thrombotic thrombocytopenic purpura. Nature 2001;413:488-494.

5 Zheng X, Chung D, Takayama TK, et al. Structure of von Willebrand factor-cleaving protease (ADAMTS13), a metalloprotease involved in thrombotic thrombocytopenic purpura. J Biol Chem 2001;276:41059-41063.

6 Soejima K, Mimura N, Hirashima M, et al. A novel human metalloprotease synthesized in the liver and secreted into the blood: possibly, the von Willebrand factor-cleaving protease? J Biochem (Tokyo) 2001; 130:475-480.

7 Soejima K, Matsumoto M, Kokame K, et al. ADAMTS13 cysteine-rich/spacer domains are functionally essential for von Willebrand factor cleavage. Blood 2003; 102:3232-3237.

8 Majerus EM, Zheng X, Tuley EA, et al. Cleavage of the ADAMTS13 propeptide is not required for protease activity. J Biol Chem 2003;278:46643-46648.

9 Apte SS. A disintegrin-like and metalloprotease (reprolysin type) with thrombospondin type 1 motifs: the ADAMTS family. Int J Biochem Cell Biol 2004;36: 981-985.

10 Tsai HM. High titers of inhibitors of von Willebrand factor-cleaving metalloproteinase in a fatal case of acute thrombotic thrombocytopenic purpura. Am J Hematol 2002;65:251-255.

11 Mannucci PM, Canciani MT, Forza I, et al. Changes in health and disease of the metalloprotease that cleaves von Willebrand factor. Blood 2002;98:2730-2735.

12 Bianchi V, Robles R, Alberio L, et al. Von Willebrand factor-cleaving protease (ADAMTS13) in thrombocytopenic disorders: a severely deficient activity is specific for thrombotic thrombocytopenic purpura. Blood 2002;100:710-713.

13 Tripodi A, Chantarangkul V, Bohm M, et al. Measurement of von Willebrand factor cleaving protease (ADAMTS-13): results of an international collaborative study involving 11 methods testing the same set of coded plasmas. J Thromb Haemost 2004;2:1601-1609.

14 Tomihara-Newberger C, Haub O, Lee HG, et al. The amn gene product is required in extraembryonic tissues for the generation of middle primitive streak derivatives. Dev Biol 1998;204:34-54.

15 Greenwel P, Schwartz M, Rosas M, et al. Characterization of fat-storing cell lines derived from normal and CCl4-cirrhotic livers. Differences in the production of interleukin-6. Lab Invest 1991;65:644-653.

16 Greenwel P, Rubin J, Schwartz M, et al. Liver fatstoring cell clones obtained from a CCl4-cirrhotic rat are heterogeneous with regard to proliferation, expression of extracellular matrix components, interleukin-6, and connexin 43. Lab Invest 1993;69:210-216.

17 Schnabl B, Choi YH, Olsen JC, et al. Immortal activated human hepatic stellate cells generated by ectopic telomerase expression. Lab Invest 2002;82:323-333.

18 Gupta S, Chowdhury NR, Jagtiani R, et al. A novel system for transplantation of isolated hepatocytes utilizing HBsAg-producing transgenic donor cells. Transplantation 1990;50:472-475.

19 Ott M, Rajvanshi P, Sokhi RP, et al. Differentiationspecific regulation of transgene expression in a diploid epithelial cell line derived from the normal F344 rat liver. J Pathol 1999;187:365-373.

20 Tsai HM, Chandler WL, Sarode R, et al. von Willebrand factor and von Willebrand factor-cleaving metalloprotease activity in Escherichia coli O157:H7-associated hemolytic uremic syndrome. Pediatr Res 2001;49: 653-659.

21 Zhou W, Tsai HM. An enzyme immunoassay of ADAMTS13 distinguishes patients with thrombotic thrombocytopenic purpura from normal individuals and carriers of ADAMTS13 mutations. Thromb Haemost 2004;91:806-811.

22 Gerritsen HE, Robles R, Lammle B, et al. Partial amino acid sequence of purified von Willebrand factorcleaving protease. Blood 2001;98:1654-1661.

23 Banno F, Kaminaka K, Soejima K, et al. Identification of strain-specific variants of mouse Adamts13 gene encoding von Willebrand factor-cleaving protease. J Biol Chem 2004;279:30896-30903.

24 Suzuki M, Murata M, Matsubara Y, et al. Detection of von Willebrand factor-cleaving protease (ADAMTS-13) in human platelets. Biochem Biophys Res Commun 2004;313:212-216.

25 Friedman SL. Stellate cells: a moving target in hepatic fibrogenesis. Hepatology 2004;40:1041-1043.

26 Bataller R, Brenner DA. Hepatic stellate cells as a target for the treatment of liver fibrosis. Semin Liver Dis 2001;21:437-451. 